Pacific

Journal of

Mathematics

THE ESSENTIAL NORM OF A COMPOSITION OPERATOR ON BLOCH SPACES

ALFonso Montes-RodríGuez 


\title{
THE ESSENTIAL NORM OF A COMPOSITION OPERATOR ON BLOCH SPACES
}

\author{
Alfonso Montes-Rodríguez
}

\begin{abstract}
We express the essential norm of a composition operator on the Bloch space and the little Bloch space as the asymptotic upper bound of a quantity involving the inducing map and the Pick-Schwarz Lemma. As a consequence, we obtain a new proof of a recently obtained characterization of the compact composition operators on Bloch spaces.
\end{abstract}

\section{Introduction.}

Let $\mathbb{D}$ denote the unit disk in the complex plane. A function $f$ analytic on the unit disk is said to belong to the Bloch space $\mathcal{B}$ if

$$
\sup _{\mathbb{D}}\left(1-|z|^{2}\right)\left|f^{\prime}(z)\right|<\infty
$$

and to the little Bloch space $\mathcal{B}_{0}$ if

$$
\lim _{|z| \rightarrow 1^{-}}\left(1-|z|^{2}\right)\left|f^{\prime}(z)\right|=0 .
$$

It is well known and easy to prove that $\mathcal{B}$ is a Banach space under the norm

$$
\|f\|=|f(0)|+\sup _{\mathbb{D}}\left(1-|z|^{2}\right)\left|f^{\prime}(z)\right|
$$

and that $\mathcal{B}_{0}$ is a closed subspace of $\mathcal{B}$. Good sources for results and references about Bloch functions are the papers of Anderson-Clunie-Pommerenke $[\mathbf{A C P}]$, Fernández $[\mathbf{F e}]$, Pommerenke $[\mathbf{P o}]$, and the book of Zhu $[\mathbf{Z h}$, Chapter 5].

If $\varphi$ is an analytic function on $\mathbb{D}$ with $\varphi(\mathbb{D}) \subset \mathbb{D}$, then the equation $C_{\varphi} f$ $=f \circ \varphi$ defines a composition operator $C_{\varphi}$ on the space of all holomorphic functions on $\mathbb{D}$. The Pick-Schwarz Lemma (see [CM, p. 47], for instance) asserts that

$$
\frac{1-|z|^{2}}{1-|\varphi(z)|^{2}}\left|\varphi^{\prime}(z)\right| \leq 1
$$


As noticed in $[\mathbf{M M}]$ this and the chain rule give an easy proof of the fact that $C_{\varphi}$ acts boundedly on the Bloch space. In fact we have

$$
\begin{aligned}
\left(1-|z|^{2}\right)\left|(f \circ \varphi)^{\prime}(z)\right| & =\left(1-|z|^{2}\right)\left|f^{\prime}(\varphi(z))\right|\left|\varphi^{\prime}(z)\right| \\
& =\frac{1-|z|^{2}}{1-|\varphi(z)|^{2}}\left|\varphi^{\prime}(z)\right|\left(1-|\varphi(z)|^{2}\right)\left|f^{\prime}(\varphi(z))\right| \\
& \leq \sup _{\mathbb{D}}\left(1-|\varphi(z)|^{2}\right)\left|f^{\prime}(\varphi(z))\right| \\
& =\sup _{\varphi(\mathbb{D})}\left(1-|w|^{2}\right)\left|f^{\prime}(w)\right| \\
& \leq \sup _{\mathbb{D}}\left(1-|z|^{2}\right)\left|f^{\prime}(z)\right| .
\end{aligned}
$$

In addition, if $C_{\varphi}$ acts boundedly on $\mathcal{B}_{0}$ then $\varphi$ must belong to $\mathcal{B}_{0}$. This follows from the fact that $C_{\varphi} z=\varphi$. Conversely, if $\varphi \in \mathcal{B}_{0}$, then from the estimates above it is easy to show that $\varphi$ induces a continuous operator on $\mathcal{B}_{0}$ (see $\left.[\mathbf{M M}]\right)$. The main goal of this paper is to compute the essential norm of $C_{\varphi}$ in terms of an asymptotic bound involving the quantity

$$
\frac{1-|z|^{2}}{1-|\varphi(z)|^{2}}\left|\varphi^{\prime}(z)\right|
$$

We recall that the essential norm of a continuous linear operator $T$ is the distance from $T$ to the compact operators, that is,

$$
\|T\|_{e}=\inf \{\|T-K\|: K \text { is compact }\} .
$$

Notice that $\|T\|_{e}=0$ if and only if $T$ is compact, so that estimates on $\|T\|_{e}$ lead to conditions for $T$ to be compact. Thus we will obtain a different proof of a recent result of Madigan and Matheson $[\mathbf{M M}]$ in which they characterize those $\varphi$ which induces compact composition operators on $\mathcal{B}$ and $\mathcal{B}_{0}$. The fundamental ideas of the proof are those used by J.H. Shapiro [Sh1] to obtain the essential norm of a composition operator on Hilbert spaces of analytic functions (Hardy and weighted Bergman spaces) in terms of natural counting functions associated with $\varphi$. However, since neither $\mathcal{B}$ nor $\mathcal{B}_{0}$ are Hilbert spaces our method differs in some interesting details from those of Shapiro.

Before going further, we want to say a word about the well-known heuristic principle which states that if a "big-oh" condition describes a class of bounded operators, then the corresponding "little-oh" condition picks out the subclass of compact operators. An excellent example of this principle in action can be seen in the paper of J.H. Shapiro [Sh1] mentioned above. The "big-oh" condition on Bloch spaces is given by (1). Madigan and Matheson were able to prove the "little-oh" condition, that is, that a composition 
operator $C_{\varphi}$ on $\mathcal{B}_{0}$ is compact if and only if

$$
\lim _{|z| \rightarrow 1^{-}} \frac{1-|z|^{2}}{1-|\varphi(z)|^{2}}\left|\varphi^{\prime}(z)\right|=0 .
$$

They also obtained (with a different proof) that $C_{\varphi}$ is compact on $\mathcal{B}$ if and only if for every $\varepsilon>0$ there exists $r, 0<r<1$, such that

$$
\sup _{|\varphi(z)|>r} \frac{1-|z|^{2}}{1-|\varphi(z)|^{2}}\left|\varphi^{\prime}(z)\right|<\varepsilon .
$$

As we will see later the conditions of compactness on $\mathcal{B}$ and $\mathcal{B}_{0}$ are actually the same. In fact, the essential norm of a composition operator is independent of the underlying space $\mathcal{B}$ or $\mathcal{B}_{0}$. This should not cause any surprise. The fact that $\mathcal{B}$ is isometrically isomorphic to the second dual of $\mathcal{B}_{0}$ and the inclusion $\mathcal{B}_{0} \subset \mathcal{B}$ corresponds to the canonical imbedding of $\mathcal{B}_{0}$ into $\mathcal{B}_{0}^{\star \star}$ (see $[\mathbf{A C P}]$ ) does not affect the computation of the essential norm. This is exactly what happens if we consider a bounded diagonal operator defined by a bounded sequence $\left\{a_{n}\right\}$ on the sequence spaces $l^{\infty}$ and $c_{0}$, respectively. Then its essential norm equals $\lim \sup a_{n}$ and this quantity is independent of the underlying space. In fact the proof of the main result in the following section is done simultaneously for both $\mathcal{B}$ and $\mathcal{B}_{0}$.

Before proceeding further, the author would like to thank Nigel J. Kalton who indicated the proof of Proposition 2.3. The author would also like to thank Joel H. Shapiro for providing the proof of Theorem 2.5, some references and helpful comments.

\section{Main result.}

Main Theorem 2.1. Suppose that $C_{\varphi}$ defines a continuous operator on $\mathcal{B}$ (or on $\left.\mathcal{B}_{0}\right)$. Then

$$
\left\|C_{\varphi}\right\|_{e}=\lim _{s \rightarrow 1^{-}} \sup _{|\varphi(z)|>s} \frac{1-|z|^{2}}{1-|\varphi(z)|^{2}}\left|\varphi^{\prime}(z)\right| .
$$

In particular, $C_{\varphi}$ is compact on $\mathcal{B}\left(\right.$ or $\left.\mathcal{B}_{0}\right)$ if and only if

$$
\lim _{s \rightarrow 1^{-}} \sup _{|\varphi(z)|>s} \frac{1-|z|^{2}}{1-|\varphi(z)|^{2}}\left|\varphi^{\prime}(z)\right|=0 .
$$

It is understood that if $\{z:|\varphi(z)|>s\}$ is the empty set for some $0<s<1$ the supremum equals zero. This happens when $\varphi(\mathbb{D})$ is a relatively compact subset of $\mathbb{D}$ and in this case it is easy to show that $C_{\varphi}$ is a compact operator.

If $\varphi$ has an angular derivative at a point $\xi \in \partial \mathbb{D}$, then we can apply the Julia Carathéodory Theorem (see [Sh2, p. 57]) and the Pick-Schwarz 
Lemma to obtain

$$
1=\liminf _{z \rightarrow \xi} \frac{1-|z|^{2}}{1-|\varphi(z)|^{2}}\left|\varphi^{\prime}(z)\right| \leq \lim _{s \rightarrow 1^{-}} \sup _{|\varphi(z)|>s} \frac{1-|z|^{2}}{1-|\varphi(z)|^{2}}\left|\varphi^{\prime}(z)\right| \leq 1 .
$$

Thus, as an immediate consequence of Theorem 2.1 we have $\left\|C_{\varphi}\right\|_{e}=1$ whenever $\varphi$ has a finite angular derivative.

Before proving Theorem 2.1 let us show that for the little Bloch space $\mathcal{B}_{0}$ there is an equivalent formula in terms of another quantity. This a simple consequence of the following proposition:

Proposition 2.2. Suppose that $C_{\varphi}$ defines a continuous operator on $\mathcal{B}_{0}$. Then

$$
\lim _{s \rightarrow 1^{-}} \sup _{|\varphi(z)|>s} \frac{1-|z|^{2}}{1-|\varphi(z)|^{2}}\left|\varphi^{\prime}(z)\right|=\limsup _{|z| \rightarrow 1^{-}} \frac{1-|z|^{2}}{1-|\varphi(z)|^{2}}\left|\varphi^{\prime}(z)\right| .
$$

Proof. As remarked in the introduction the fact that $C_{\varphi}$ acts boundedly on $\mathcal{B}_{0}$ implies that $\varphi \in \mathcal{B}_{0}$. If $\varphi(\mathbb{D})$ is a relatively compact subset of $\mathbb{D}$, then both limits in $(2)$ are zero and coincide. So we may suppose that $\varphi(\mathbb{D})$ is not a relatively compact subset of $\mathbb{D}$. Let $0<s_{n}<1$ be any increasing sequence tending to 1 . We set $t_{n}=\inf \left\{t:|\varphi(z)|>s_{n}\right.$ for some $z$ with $\left.|z|>t\right\}$. By continuity $\left\{t_{n}\right\}$ also tends to 1 . Since $\left\{z:|z|>t_{n}\right\}=\{z:|\varphi(z)|>$ $s_{n}$ and $\left.|z|>t_{n}\right\} \cup\left\{z:|\varphi(z)| \leq s_{n}\right.$ and $\left.|z|>t_{n}\right\}$ we find that the left hand side of (2) is less than or equal to the right hand side of (2). On the other hand, we can always find a sequence $\left\{z_{n}\right\}$ for which

$$
\begin{aligned}
\lim _{n \rightarrow \infty} \frac{1-\left|z_{n}\right|^{2}}{1-\left|\varphi\left(z_{n}\right)\right|^{2}}\left|\varphi^{\prime}\left(z_{n}\right)\right| & =\lim _{s \rightarrow 1^{-}} \sup _{|z|>s} \frac{1-|z|^{2}}{1-|\varphi(z)|^{2}}\left|\varphi^{\prime}(z)\right| \\
& =\limsup _{|z| \rightarrow 1^{-}} \frac{1-|z|^{2}}{1-|\varphi(z)|^{2}}\left|\varphi^{\prime}(z)\right| .
\end{aligned}
$$

Then either there is a subsequence $\left\{z_{n_{k}}\right\}$ such that $\left\{\left|\varphi\left(z_{n_{k}}\right)\right|\right\} \rightarrow 1$ as $k \rightarrow \infty$, or for every positive integer $n$ we have $\left|\varphi\left(z_{n}\right)\right| \leq s_{0}$ for some $0<s_{0}<1$. Clearly, in the former case both limits in (2) coincide. For the latter case we find that the limit in (3) is zero because $\varphi \in \mathcal{B}_{0}$. Since this limit is greater than or equal to the limit on the left hand side of (2), we find that they are the same again. The proof is now finished.

Now we turn to the proof of our main result.

The lower estimate. First we show that:

$$
\left\|C_{\varphi}\right\|_{e} \geq \lim _{s \rightarrow 1^{-}} \sup _{|\varphi(z)| \geq s} \frac{1-|z|^{2}}{1-|\varphi(z)|^{2}}\left|\varphi^{\prime}(z)\right| .
$$

Instead of the reproducing kernels used by Shapiro for the Hardy and Bergman spaces we will use the sequence $\left\{z^{n}\right\}_{n \geq 2}$. This sequence converges 
uniformly on compact subsets of the unit disk. An elementary computation shows that

$$
\left\|z^{n}\right\|=\max _{\mathbb{D}}\left(1-|z|^{2}\right)\left|n z^{n-1}\right|=\frac{2 n}{n+1}\left(\frac{n-1}{n+1}\right)^{(n-1) / 2} .
$$

Observe that for each $n \geq 2$ the above maximum is attained at any point on the circle centered at the origin and of radius $r_{n}=\left(\frac{n-1}{n+1}\right)^{1 / 2}$. These maxima form a decreasing sequence which tends to $2 / e$.

Therefore, the sequence $\left\{z^{n}\right\}_{n \geq 2}$ is bounded away from zero. Now we consider the normalized sequence $\left\{f_{n}=\frac{z^{n}}{\left\|z^{n}\right\|}\right\}$ which also tends to zero uniformly on compact subsets of the unit disk. For each $n \geq 2$ we define the closed annulus $A_{n}=\left\{z \in \mathbb{D}: r_{n} \leq|z| \leq r_{n+1}\right\}$ and compute

$$
\begin{aligned}
\min _{A_{n}}\left(1-|z|^{2}\right)\left|f_{n}^{\prime}(z)\right| & =\left(1-r_{n+1}^{2}\right)\left|f_{n}^{\prime}\left(r_{n+1}\right)\right| \\
& =\left(\frac{n+1}{n+2}\right)\left(\frac{n^{2}+n}{n^{2}+n-2}\right)^{(n-1) / 2} .
\end{aligned}
$$

Observe that these minima tend to 1 as $n \rightarrow \infty$ and for each $n \geq 2$ the minimum above is attained at any point of the circle centered at the origin and of radius $r_{n+1}$. For the moment fix any compact operator $K$ on $\mathcal{B}_{0}$ or $\mathcal{B}$. The uniform convergence on compact subsets of the sequence $\left\{f_{n}\right\}$ to zero and the compactness of $K$ imply that $\left\|K f_{n}\right\| \rightarrow 0$. It is easy to show that if a bounded sequence that is contained in $\mathcal{B}_{0}$ converges uniformly on compact subsets of the unit disk, then it also converges weakly to zero in $\mathcal{B}_{0}$ as well as in $\mathcal{B}$. Thus

$$
\begin{aligned}
\left\|C_{\varphi}-K\right\| & \geq \limsup _{n}\left\|\left(C_{\varphi}-K\right) f_{n}\right\| \\
& \geq \limsup _{n}\left(\left\|C_{\varphi} f_{n}\right\|-\left\|K f_{n}\right\|\right) \\
& =\limsup _{n}\left\|C_{\varphi} f_{n}\right\| .
\end{aligned}
$$

Upon taking the infimum of both sides of this inequality over all compact operators $K$, we obtain the lower estimate:

$$
\begin{aligned}
\left\|C_{\varphi}\right\|_{e} & \geq \limsup _{n}\left\|C_{\varphi} f_{n}\right\| \\
& =\limsup _{n} \sup _{\mathbb{D}}\left(1-|z|^{2}\right)\left|f_{n}^{\prime}(\varphi(z))\right|\left|\varphi^{\prime}(z)\right| \\
& =\limsup _{n} \sup _{\mathbb{D}} \frac{1-|z|^{2}}{1-|\varphi(z)|^{2}}\left|\varphi^{\prime}(z)\right|\left(1-|\varphi(z)|^{2}\right)\left|f_{n}^{\prime}(\varphi(z))\right| .
\end{aligned}
$$

Now (6) is greater than or equal to

$$
\limsup _{n} \sup _{\varphi(z) \in A_{n}} \frac{1-|z|^{2}}{1-|\varphi(z)|^{2}}\left|\varphi^{\prime}(z)\right|\left(1-|\varphi(z)|^{2}\right)\left|f_{n}^{\prime}(\varphi(z))\right|
$$


and $(7)$ is greater than or equal to

(8) $\quad \limsup _{n} \sup _{\varphi(z) \in A_{n}} \frac{1-|z|^{2}}{1-|\varphi(z)|^{2}}\left|\varphi^{\prime}(z)\right| \min _{\varphi(z) \in A_{n}}\left(1-|\varphi(z)|^{2}\right)\left|f_{n}^{\prime}(\varphi(z))\right|$.

If $\varphi(\mathbb{D})$ is a relatively compact subset of $\mathbb{D}$ both sides of $(4)$ are zero and there is nothing to prove. Otherwise we find that $\min _{\varphi(z) \in A_{n}}\left(1-|\varphi(z)|^{2}\right)\left|f_{n}^{\prime}(\varphi(z))\right|$ $=\min _{A_{n}}\left(1-|z|^{2}\right)\left|f_{n}^{\prime}(z)\right|$ because the minimum in (5) is attained at any point on the circle centered at the origin and of radius $r_{n+1}$. Since these minima tend to 1 as $n \rightarrow \infty$, it follows that (8) is equal to

$$
\limsup _{n} \sup _{\varphi(z) \in A_{n}} \frac{1-|z|^{2}}{1-|\varphi(z)|^{2}}\left|\varphi^{\prime}(z)\right| \text {. }
$$

Finally, an easy exercise shows that (9) coincides with the right hand side of (4).

To obtain the upper estimate in the case of the Hardy and Bergman spaces, Shapiro [Sh1] used the operators $P_{n}$ which take $f$ to the $n$th partial sum of its Taylor series. On the Hardy space these operators satisfy: i) Each $P_{n}$ is compact, ii) $\left(I-P_{n}\right) f$ tends to zero uniformly on compact subsets for any $f$ in the Hardy space, and iii) for each $n$ the norm in the Hardy space of $I-P_{n}$ equals 1 . Although each $P_{n}$ is also compact in the Bloch space, and $\left(I-P_{n}\right) f$ tends to zero uniformly on compact subsets for each function $f \in \mathcal{B}$, this sequence does not satisfy anything analogous to iii) above. In fact, $\left\|P_{n}\right\| \geq C \log n$ where $C$ is a universal constant (see [ACP, p. 18-19]). Therefore, by the reverse triangle inequality $\left\|I-P_{n}\right\| \geq C \log n-1$. One of the issues here is that in general it is not easy to compute exactly either the norms of Bloch functions, or the norms of operators defined on Bloch spaces.

To obtain the upper estimate we need the operators $K_{n}, n \geq 2$, which take each function $f(z)$ to $f\left(\frac{n-1}{n} z\right)$. Every operator $K_{n}$ is compact on $\mathcal{B}$ (or $\left.\mathcal{B}_{0}\right)$. We also have that $\left(I-K_{n}\right) f$ tends to zero uniformly on compact subsets of the unit disk for every $f \in \mathcal{B}$, and (although we do not know if $\lim _{n \rightarrow \infty}\left\|I-K_{n}\right\|=1$ ) we have the following proposition, whose proof is delayed.

Proposition 2.3. There exists a sequence of convex combinations $L_{n}$ of $K_{n}\left(L_{n}=\sum_{m \geq n} c_{n, m} K_{m}\right.$ with $c_{m, n}>0$ and $\left.\sum_{m \geq n} c_{n, m}=1\right)$ such that $\lim _{n \rightarrow \infty}\left\|I-L_{n}\right\|=1$.

The upper estimate. The goal now is to show that

$$
\left\|C_{\varphi}\right\|_{e} \leq \lim _{s \rightarrow 1^{-}} \sup _{|\varphi(z)|>s} \frac{1-|z|^{2}}{1-|\varphi(z)|^{2}}\left|\varphi^{\prime}(z)\right| .
$$


This will be accomplished by applying Proposition 2.3. Since each $L_{n}$ is compact so is $C_{\varphi} L_{n}$. Therefore

$$
\left\|C_{\varphi}\right\|_{e} \leq\left\|C_{\varphi}-C_{\varphi} L_{n}\right\|=\left\|C_{\varphi}\left(I-L_{n}\right)\right\| .
$$

On the other hand, we have

$$
\begin{aligned}
& \left\|C_{\varphi}\left(I-L_{n}\right)\right\| \\
& =\sup _{\|f\|=1}\left\|C_{\varphi}\left(I-L_{n}\right) f\right\| \\
& =\sup _{\|f\|=1|z|<1} \sup _{\left.|z|<\left.\right|^{2}\right)\left|\left(\left(I-L_{n}\right) f\right)^{\prime}(\varphi(z))\right|\left|\varphi^{\prime}(z)\right|} \\
& =\sup _{\|f\|=1} \sup _{|z|<1} \frac{1-|z|^{2}}{1-|\varphi(z)|^{2}}\left|\varphi^{\prime}(z)\right|\left(1-|\varphi(z)|^{2}\right)\left|\left(\left(I-L_{n}\right) f\right)^{\prime}(\varphi(z))\right| .
\end{aligned}
$$

Now fix $0<s<1$. Then the right hand side of (11) is less than or equal to

$$
\begin{aligned}
& \sup _{\|f\|=1} \sup _{|\varphi(z)| \leq s} \frac{1-|z|^{2}}{1-|\varphi(z)|^{2}}\left|\varphi^{\prime}(z)\right|\left(1-|\varphi(z)|^{2}\right)\left|\left(\left(I-L_{n}\right) f\right)^{\prime}(\varphi(z))\right| \\
& \quad+\sup _{\|f\|=1} \sup _{|\varphi(z)|>s} \frac{1-|z|^{2}}{1-|\varphi(z)|^{2}}\left|\varphi^{\prime}(z)\right|\left(1-|\varphi(z)|^{2}\right)\left|\left(\left(I-L_{n}\right) f\right)^{\prime}(\varphi(z))\right| .
\end{aligned}
$$

By applying the Pick-Schwarz Lemma in the first term, and the fact that for $f$ in the unit ball

$$
\begin{aligned}
& \sup _{|\varphi(z)|>s}\left(1-|\varphi(z)|^{2}\right)\left|\left(\left(I-L_{n}\right) f\right)^{\prime}(\varphi(z))\right| \\
& \leq \sup _{|z|<1}\left(1-|z|^{2}\right)\left|\left(\left(I-L_{n}\right) f\right)^{\prime}(z)\right| \leq\left\|I-L_{n}\right\|
\end{aligned}
$$

to the second term, we find that (12) is less than or equal to

$$
\begin{aligned}
& \sup _{\|f\|=1|w| \leq s} \sup _{|w| \leq}\left(1-|w|^{2}\right)\left|\left(\left(I-L_{n}\right) f\right)^{\prime}(w)\right| \\
& \quad+\left\|I-L_{n}\right\| \sup _{|\varphi(z)|>s} \frac{1-|z|^{2}}{1-|\varphi(z)|^{2}}\left|\varphi^{\prime}(z)\right| .
\end{aligned}
$$

Let us prove that the first term in (13) tends to zero as $n \rightarrow \infty$. By the triangle inequality we have that the first term in (13) is less than or equal to

$$
\sum_{m \geq n} c_{n, m} \sup _{\|f\|=1} \sup _{|w| \leq s}\left(1-|w|^{2}\right)\left|\left(\left(I-K_{m}\right) f\right)^{\prime}(w)\right| .
$$


By the triangle inequality again we find that $\left(1-|w|^{2}\right)\left|\left(\left(I-K_{m}\right) f\right)^{\prime}(w)\right|$ is less than or equal to

$$
\begin{aligned}
\sup _{\|f\|=1} \sup _{|w| \leq s}\left(1-|w|^{2}\right) \mid & f^{\prime}(w)-f^{\prime}\left(\left(1-\frac{1}{m}\right) w\right) \mid \\
& +\frac{1}{m} \sup _{\|f\|=1|w| \leq s} \sup _{|| w \mid}\left(1-|w|^{2}\right)\left|f^{\prime}\left(\left(1-\frac{1}{m}\right) w\right)\right| .
\end{aligned}
$$

By integrating $f^{\prime \prime}$ along the radial segment $[(1-1 / m) w, w]$ it is easy to see that the first term in (15) is less than or equal to

$$
\frac{1}{m} \sup _{\|f\|=1} \sup _{|w| \leq s}\left(1-|w|^{2}\right)|w|\left|f^{\prime \prime}(\xi(w))\right|
$$

where $\xi(w)$ belongs to the radial segment $[(1-1 / m) w, w]$ that is still contained in the closed disk of radius $s$. The Cauchy inequalities applied to a circle $C(\xi(w))$ centered at $\xi(w)$ and of any fix radius $0<R<1-s$ yields that (16) is less than or equal to

$$
\frac{1}{m R} \sup _{\|f\|=1|w| \leq s} \sup _{|w|}\left(1-|w|^{2}\right)|w| \max _{|z|=s+R}\left|f^{\prime}(z)\right| .
$$

On the other hand, on the unit ball of $\mathcal{B}\left(\right.$ or $\mathcal{B}_{0}$ ) we have $\max _{|z|=s+R}\left|f^{\prime}(z)\right| \leq$ $\frac{1}{1-(s+R)^{2}}$. So we find that $(17)$ is less than or equal to

$$
\frac{1}{m R} \sup _{|w| \leq s}\left(1-|w|^{2}\right)|w| \frac{1}{1-(s+R)^{2}} \leq \frac{1}{m R} \frac{s}{1-(s+R)^{2}} .
$$

Since the second term in (15) is less than $1 / m$ we find that (15) is $\leq C / m$, where $C$ only depends on $s$. Therefore, we find that (14) is less than or equal to

$$
\sum_{m \geq n} c_{n, m} \frac{C}{m} \leq \sum_{m \geq n} c_{n, m} \frac{C}{n}=\frac{C}{n}
$$

which tends to zero as $n \rightarrow \infty$. Hence, letting $n \rightarrow \infty$ in (13), applying Proposition 2.3 and putting everything together, the following inequality follows

$$
\left\|C_{\varphi}\right\|_{e} \leq \sup _{|\varphi(z)|>s} \frac{1-|z|^{2}}{1-|\varphi(z)|^{2}}\left|\varphi^{\prime}(z)\right| .
$$

Since $s$ was arbitrary inequality (10) holds.

Remarks. 1. By the triangle inequality we have $\left\|I-K_{n}\right\| \leq\|I\|+\left\|K_{n}\right\|=2$. Therefore, if we use the sequence $\left\{K_{n}\right\}$ instead of the sequence $\left\{L_{n}\right\}$ in the proof of the upper estimate, then we obtain twice the upper estimate. However, that is enough to characterize the compact composition operators on Bloch spaces without requiring Proposition 2.3. 
2. Note that it is not possible to have a sequence $\left\{L_{n}\right\}$ of convex combinations of $\left\{K_{n}\right\}_{m \geq n}$ such that $\lim \left\|I-L_{n}\right\|<1$. For if this were the case, we could obtain an upper estimate strictly less than the lower estimate, a contradiction. Thus in order to prove Proposition 2.3 it is enough to construct a sequence $\left\{L_{n}\right\}$ of convex combinations of $\left\{K_{m}\right\}_{m \geq n}$ such that $\lim \sup _{n \rightarrow \infty}\left\|I-L_{n}\right\| \leq 1$.

The proof of Theorem 2.1 will be completed once we have proved Proposition 2.3. In order to do this we need some basic facts about Bloch spaces. Recall that dual space $\mathcal{B}_{0}^{\star}$ of $\mathcal{B}_{0}$ is isomorphic to the space $A^{1}(\mathbb{D})$ of analytic functions on the unit disk such that

$$
\int_{\mathbb{D}}|g(z)| d A(z)<\infty
$$

where $d A(z)$ is Lebesgue area measure on $\mathbb{D}$, normalized to have total mass 1 , that is, $d A(z)=\frac{1}{\pi} d x d y=\frac{1}{\pi} \rho d \theta d \rho$ for $z=x+i y=\rho e^{i \theta}$. This duality is realized by the integral pairing

$$
\langle f, g\rangle=\int_{\mathbb{D}} f(z) \overline{g(z)} d A(z)
$$

(see [Zh, p. 87]). Let $0<r<1$ be fixed and let $K_{r}: \mathcal{B}_{0} \rightarrow \mathcal{B}_{0}$ be the operator which assigns to each function $f$ the function $f(r z)$. Now, for any $f(z)=\sum_{n=0}^{\infty} a_{n} z^{n} \in \mathcal{B}_{0}$ and any $g(z)=\sum_{n=0}^{\infty} b_{n} z^{n} \in A^{1}(\mathbb{D})$ a straightforward computation shows that

$$
\langle f(r z), g(z)\rangle=\sum_{n=0}^{\infty} \frac{r^{n}}{n+1} a_{n} \bar{b}_{n}=\langle f(z), g(r z)\rangle .
$$

Thus, the adjoint operator $K_{r}^{\star}: A^{1}(\mathbb{D}) \rightarrow A^{1}(\mathbb{D})$ acts in the same way as does $K_{r}$. We also have that the Bloch space $\mathcal{B}$ is the dual of $A^{1}(\mathbb{D})$ under the same integral pairing. Thus in a similar way, it can be shown that the bi-adjoint operator $K_{r}^{\star \star}: \mathcal{B} \rightarrow \mathcal{B}$ of $K_{r}$ is the operator that assigns to each function $f(z)$ the function $f(r z)$. Thus, a little abuse of the language allows us denote $K_{r}^{\star}$ and $K_{r}^{\star \star}$ by $K_{r}$. With this we may observe that if we have constructed the sequence $\left\{L_{n}\right\}$ required by Proposition 2.3 for $\mathcal{B}_{0}$, then just considering the bi-adjoint sequence the result follows for the Bloch space $\mathcal{B}$. This is trivial because $L_{n}^{\star \star}=\left(\sum_{m \geq n} c_{n, m} K_{n}\right)^{\star \star}=\sum_{m \geq n} c_{n, m} K_{n}$ and $\left\|\left(I-L_{n}\right)^{\star \star}\right\|=\left\|I-L_{n}\right\|$.

To prove Proposition 2.3 we also need the following proposition about the compact operators $K_{r}$.

Proposition 2.4. For any $g \in A^{1}(\mathbb{D})$ we have $\left\|K_{r} g-g\right\| \rightarrow 0$ as $r \rightarrow 1^{-}$. 
Proof. Let $\varepsilon>0$ be fixed. By the continuity of the integral we can find an $s, 1>s>0$, such that

$$
\int_{|z|>s}|g(z)| d A(z)<\frac{\varepsilon}{3}
$$

Now $r s \rightarrow s$ and $1 / r \rightarrow 1$ as $r \rightarrow 1$. Therefore, the change of variables $w=r z$ and the above display show that

$$
\begin{aligned}
\int_{|z|>s}|g(r z)| d A(z) & =\frac{1}{r} \int_{r s<|w| \leq r}|g(w)| d A(w) \\
& \leq \frac{1}{r} \int_{r s<|w|}|g(w)| d A(w)<\frac{\varepsilon}{3}
\end{aligned}
$$

for $r$ near enough to 1 . On the other hand, since $K_{r} g$ tends to $g$ uniformly on compact subsets of the unit disk as $r \rightarrow 1^{-}$, we have $\max _{|z| \leq s}|g(r z)-g(z)|<$ $\varepsilon / 3$ for $r$ near enough to 1 . Thus for $r$ close to 1 we have

$$
\begin{aligned}
\|g(r z)-g(z)\| & =\int_{|z| \leq s}|g(r z)-g(z)| d A(z)+\int_{|z|>s}|g(r z)-g(z)| d A(z) \\
& <\frac{\varepsilon}{3} s^{2}+\int_{|z|>s}|g(z)| d A(z)+\int_{|z|>s}|g(r z)| d A(z) \\
& <\frac{\varepsilon}{3}+\frac{\varepsilon}{3}+\frac{\varepsilon}{3} \\
& =\varepsilon .
\end{aligned}
$$

Since $\varepsilon$ was arbitrary, the result follows.

Given two Banach spaces $X$ and $Y$ we denote by $\mathcal{L}(X, Y)$ the Banach space of bounded operators from $X$ into $Y$ and by $\mathcal{K}(X, Y)$ the Banach space of compact operators from $X$ into $Y$. We need a theorem of Mazur that asserts that if a sequence in a Banach space converges weakly, then some sequence of convex combinations converges in norm (see [Di, 15]). We begin with the following theorem, whose proof was provided by Joel H. Shapiro (alternatively, in the proof of Proposition 2.3, we can use Theorem 1 in $[\mathbf{K a} \mathbf{1}])$.

Theorem 2.5. Suppose $X$ and $Y$ are Banach spaces and $\left\{T_{n}\right\}$ is a sequence of compact linear operators from $X$ to $Y$. Suppose further that for every $y^{\star} \in Y^{\star}$ and $x^{\star \star} \in X^{\star \star}$ we have: $\left\langle T_{n}^{\star} y^{\star}, x^{\star \star}\right\rangle \rightarrow 0$. Then there is a sequence $\left\{S_{n}\right\}$ of convex combinations of the original $T_{n}$ such that $\left\|S_{n}\right\| \rightarrow 0$.

Proof. Let $Q$ denote the cartesian product of the closed unit ball of $Y^{\star}$ and the closed unit ball of $X^{\star \star}$, where each ball has its respective weak star topology. Thus $Q$ is a compact Hausdorff space. For $T \in \mathcal{K}(X, Y)$ the function $\widehat{T^{\star}}: Q \rightarrow \mathbb{C}$ defined by:

$$
\widehat{T^{\star}}\left(\left(y^{\star}, x^{\star \star}\right)\right)=\left\langle T^{\star} y^{\star}, x^{\star \star}\right\rangle \quad\left(=x^{\star \star}\left(T^{\star}\left(y^{\star}\right)\right)\right) \quad\left(x^{\star \star} \in X^{\star \star} \text { and } y^{\star} \in Y^{\star}\right)
$$


belongs to $C(Q)$ (see [Ka1, Lemma 1]), and the map $T^{\star} \rightarrow \widehat{T^{\star}}$ is an isometry taking a certain closed subspace of $\mathcal{K}\left(Y^{\star}, X^{\star}\right)$ (namely the weak-star continuous compacts) onto a closed subspace of $C(Q)$. By this correspondence and the Hahn-Banach theorem, $T_{n}^{\star} \rightarrow 0$ weakly in $\mathcal{L}\left(Y^{\star}, X^{\star}\right)$ if and only if $\widehat{T_{n}^{\star}}$ tends weakly in $C(Q)$.

By the Riesz Representation Theorem and the Lebesgue bounded convergence theorem, a sequence of functions in $C(Q)$ converges weakly to zero if and only if it converges pointwise to zero. But the hypothesis on $\left\{T_{n}^{\star}\right\}$ is just the statement that $\widehat{T_{n}^{\star}} \rightarrow 0$ pointwise on $Q$. In addition, it follows from the Uniform Boundedness Principle that $\sup _{n}\left\|T_{n}\right\|<\infty$, hence because $\left\|T_{n}\right\|=\left\|T_{n}^{\star}\right\|$, the sequence $\widehat{T_{n}^{\star}}$ is also bounded. Thus $T_{n}^{\star} \rightarrow 0$ weakly in $\mathcal{L}\left(Y^{\star}, X^{\star}\right)$ and so by Mazur's theorem, there is a sequence of convex combinations $\left\{S_{n}^{\star}\right\}$ of the original operators $\left\{T_{n}^{\star}\right\}$, such that $\left\|S_{n}^{\star}\right\| \rightarrow 0$. Thus also $\left\|S_{n}\right\| \rightarrow 0$, which is the desired result.

To prove Proposition 2.3 we will use the fact that $\mathcal{B}_{0}$ is isomorphic to the sequence space $c_{0}$. For completeness we include a proof of this fact. Let us consider the function $\phi(r)=1-r^{2}$ defined on the interval [0,1] and let $h_{\infty}(\phi)$ be the Banach space of complex-valued functions, $u$ harmonic in the unit disk with the norm

$$
\|u\|_{\phi}=\sup _{\mathbb{D}}|u(z)| \phi(z)
$$

and let $h_{0}(\phi)$ be the closed subspace of functions $u$ for which $|u(z)| \phi(z) \rightarrow 0$ as $|z| \rightarrow 1^{-}$. The space $h_{0}(\phi)$ is isomorphic to the sequence space $c_{0}$ (see $\left[\mathbf{S W}\right.$, Theorem 7]). Finally, we denote by $H_{0}(\phi)$ the closed subspace of those functions in $h_{0}(\phi)$ that are analytic on the unit disk. Now, observe that $h_{0}(\phi)$ is self-conjugate, that is, $u \in h_{0}(\phi)$ if and only if its conjugate $\bar{u} \in h_{0}(\phi)$. This fact along with the Closed Graph Theorem implies that the Riesz projection $P: h_{0}(\phi) \rightarrow H_{0}(\phi)$ defined by

$$
P u=\frac{1}{2}(u+i \bar{u})+\frac{1}{2} u(0)
$$

is bounded. Thus we can express $h_{0}(\phi)=H_{0}(\phi) \oplus \operatorname{ker} P$. Now, a famous theorem of Pelczyński (see [Pe, Theorem 1]) asserts that if $F$ is a complemented subspace of $c_{0}$, then either $F$ is isomorphic to $c_{0}$ or $F$ is of finite dimension. Since $H_{0}(\phi)$ is complemented in a space isomorphic to $c_{0}$, it follows that $H_{0}(\phi)$ is isomorphic to $c_{0}$. Finally, since $H_{0}(\phi)$ is isometrically isomorphic to $\mathcal{B}_{0}$ (consider the map $f \rightarrow f^{\prime}$ ), it follows that $\mathcal{B}_{0}$ is isomorphic to $c_{0}$.

As mentioned, the following argument was indicated by N. J. Kalton. Some parts of this argument already appear in [Ka1] (see also [Ka2, Theorem 2.4] and [HWW, Chapter VI, Theorem 4.17 and Theorem 5.7]). 
Proof of Proposition 2.3. As pointed out before it is enough to prove the result for the little Bloch space. By Remark 2 above it will be sufficient to show that for any $\varepsilon>0$ there exists a convex linear combination $L_{n}$ of $\left\{K_{m}\right\}_{m \geq n}$ with $\left\|I-L_{n}\right\|<1+\varepsilon$. Once this is done the proof can be completed by a simple diagonal argument.

Since $\mathcal{B}_{0}$ is isomorphic to the sequence space $c_{0}$, James's Theorem (see [LT, p. 97]) can be applied to find that there exists a Banach subspace $X_{0} \subset c_{0}$ such that the Banach-Mazur distance from $\mathcal{B}_{0}$ to $X_{0}$ is strictly less than $\sqrt{1+\varepsilon}$. That is, there is an isomorphism $T: \mathcal{B}_{0} \rightarrow X_{0}$ such that $\|T\|\left\|T^{-1}\right\|<\sqrt{1+\varepsilon}$. We define $T_{n}=T K_{n} T^{-1}: X_{0} \rightarrow X_{0}$. Upon applying Proposition 2.4 we find that

$$
\lim _{n \rightarrow \infty}\left\|T_{n}^{\star} x^{\star}-x^{\star}\right\|=0
$$

for each $x^{\star} \in X_{0}^{\star}$. If $P_{n}$ is the sequence of coordinate projections on $c_{0}$, then we also have

$$
\lim _{n \rightarrow \infty}\left\|P_{n}^{\star} x^{\star}-x^{\star}\right\|=0
$$

for each $x^{\star} \in l^{1}=c_{0}^{\star}$ the dual space of $c_{0}$. Now, if $J$ denotes the inclusion from $X_{0}$ into $c_{0}$, then $J T_{n}-P_{n} J \in K\left(X_{0}, c_{0}\right)$. Furthermore, by applying (18) and (19) the sequence $\left\langle\left(J T_{n}-P_{n} J\right)^{\star} x^{\star}, y^{\star \star}\right\rangle$ tends to zero for $y^{\star \star} \in X_{0}^{\star \star}$ and $x^{\star} \in l^{1}$. Thus we may apply Theorem 2.5 to see that there exist a sequence of convex combinations of $\left\{J T_{n}-P_{n} J\right\}$ that tends to zero in norm. This implies that there are sequences $\left\{T_{n}^{c}\right\}$ and $\left\{P_{n}^{c}\right\}$ of convex combinations of $\left\{T_{m}\right\}_{m \geq n}$ and $\left\{P_{m}\right\}_{m \geq n}$, respectively, such that $J T_{n}^{c}-P_{n}^{c} J$ tends to zero in norm. Therefore, we have for all sufficiently large $n$ :

$$
\left\|I-T_{n}^{c}\right\|=\left\|J\left(I-T_{n}^{c}\right)\right\| \leq\left\|\left(I-P_{n}^{c}\right) J\right\|+\left\|J T_{n}^{c}-P_{n}^{c} J\right\| \leq \sqrt{1+\varepsilon},
$$

where we have used successively: The fact that $J: X_{0} \rightarrow c_{0}$ is the inclusion map, the triangle inequality, and the inequality $\left\|\left(I-P_{n}^{c}\right) J\right\| \leq 1$. Finally, if we set $L_{n}=T^{-1} T_{n}^{c} T$, then

$$
\left\|I-L_{n}\right\|=\left\|T^{-1}\left(I-T_{n}^{c}\right) T\right\| \leq\left\|T^{-1}\right\|\left\|I-T_{n}^{c}\right\|\|T\|<1+\varepsilon
$$

that is what we had to prove. The proof of Proposition 2.3, and therefore that of Theorem 2.1, is now completed.

Remark. A sequence of compact operators satisfying the required properties to get the upper estimate can also be obtained more directly by using the theory of $M$-ideals of compact operators (see [Ka2, Theorem 2.4] and [HWW, Chapter VI, Theorem 4.17]). 


\section{References}

[ACP] J.M Anderson, J. Clunie and Ch. Pommerenke, On Bloch functions and normal functions, J. Reine Angew. Math., 270 (1974), 12-37.

[CM] C. Cowen and B. MacCluer, Composition Operators on Spaces of Analytic Functions, CRC Press, 1995.

[Di] J. Diestel, Sequences and Series in Banach Spaces, Springer-Verlag, Berlin-New York, 1984.

[Fe] J.L. Fernández, On the coefficients of Bloch functions, J. London Math. Soc., 29(2) (1984), 94-102.

[HWW] P. Harmand, D. Werner and W. Werner, $M$-ideals in Banach Spaces and Banach Algebras, Springer-Verlag, Berlin-Heidelberg-New York, 1993.

[Ka1] N.J. Kalton, Spaces of compact operators, Math. Ann., 208(2) (1974), 267-278.

[Ka2] _ M-ideals of compact operators, Illinois J. Math., 37 (1993), 147-169.

[LT] J. Lindenstrauss and L. Tzafriri, Classical Banach Spaces I, Springer, BerlinHeidelberg-New York, 1977.

[MM] K. Madigan and A. Matheson, Compact composition operators on the Bloch space, Trans. Amer. Math. Soc., 347 (1995), 2679-2687.

[Pe] A. Pelczynski, Projections in certain Banach spaces, Studia Math., 19 (1960), 209-228.

[Po] Ch. Pommerenke, On Bloch functions, J. London Math. Soc., 2(2), (1970), 689685.

[Sh1] J.H. Shapiro, The essential norm of a composition operator, Annals of Math., 125 (1987), 375-404.

[Sh2] Composition Operators and Classical Function Theory, Springer-Verlag, 1993.

[SW] A.L. Shields and D.L. Williams, Bounded projections, duality, and multipliers in spaces of harmonic functions, J. Reine Angew. Math., 299/300 (1978), 256-279.

[Zh] K. Zhu, Operator Theory in Function Spaces, Marcel Dekker, I.N.C., 1990.

Received May 30, 1997 and revised May 3, 1998.

Universidad de Sevilla

SEVILla 41080

SPAIN

E-mail address: amontes@cica.es 